$$
\text { PPPL - - 3247 }
$$

\title{
Energetic Particle Physics with Applications in Fusion and Space Plasmas
}

\author{
C. Z. Cheng \\ Princeton Plasma Physics Laboratory \\ Princeton University, Princeton, New Jersey 08543
}

\begin{abstract}
Energetic particle physics is the study of the effects of energetic particles on "collective" electromagnetic (EM) instabilities and energetic particle transport in plasmas. Anomalously large energetic particle transport is often caused by low frequency MHD instabilities, which are driven by these energetic particles in the presence of a much denser background of thermal particles. The theory of collective energetic particle phenomena studies complex wave-particle interactions in which particle kinetic physics involving small spatial and fast temporal scales can strongly affect the MHD structure and long-time behavior of plasmas. The difficulty of modeling kinetic-MHD multiscale coupling processes stems from the disparate scales which are traditionally analyzed separately: the macroscale MHD phenomena are studied using the fluid MHD framework, while microscale kinetic phenomena are best described by complicated kinetic theories. We have developed a kinetic-MHD model that properly incorporates major particle kinetic effects into the MHD fluid description. For tokamak plasmas a nonvariational kinetic-MHD stability code, the NOVA-K code, has been successfully developed and applied to study problems such as the excitation of fishbone and Toroidal Alfvén Eigenmodes (TAE) and the sawtooth stabilization by energetic ions in tokamaks.
\end{abstract}

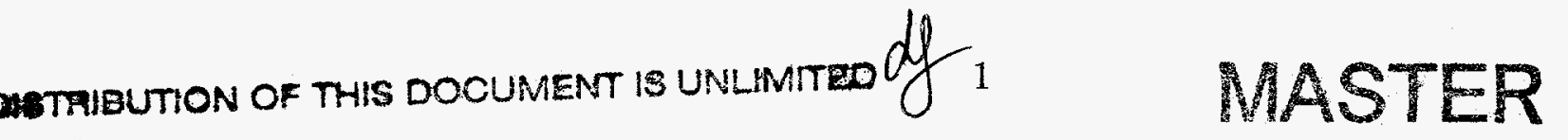




\section{DISCLAIMER}

Portions of this document may be illegible in electronic image products. Images are produced from the best available original document. 
In space plasmas we have employed the kinetic-MHD model to study the energetic particle effects on the ballooning-mirror instability which explains the multisatellite obervation of the stability and field-aligned structure of compressional Pc 5 waves in the magnetospheric ring current plasma.

\section{INTRODUCTION}

In fusion plasma physics, energetic particle physics was developed to understand the behavior of fast ions in tokamaks, particularly on their confinement, global MHD stability, and plasma heating effects. The main goal was to insure that superthermal ions (such as 3.5 $\mathrm{MeV}$ alpha particles created by Deuterium-Tritium reactions, $\mathrm{MeV}$ ion cyclotron wave heated ions, and few hundred $\mathrm{keV}$ neutral beam injected ions) were confined well enough to transfer their energy to the thermal plasma, and did not create new plasma instabilities. Major energetic particle physics theories discovered and experiments included Toroidal Alfvén Eigenmodes (TAE) [1,2] and its destabilization [3-6] by energetic particles, sawtooth (internal kink mode) stabilization $[4,6,7]$ and fishbone mode destabilization $[4,6,8]$ by energetic trapped particles. These phenomena involve low frequency multiscale kinetic-MHD phenomena in which kinetic physics involving small spatial and fast temporal scales can strongly affect the global structure and long time behavior of plasmas. Coupling between multiple spatial and temporal scales is an inherently difficult process to model. The difficulty stems from the disparate scales which traditionally are analyzed separately. Long time global-scale phenomena are generally studied using the single fluid MHD framework, while short time microscale phenomena are best described with kinetic theories. We have estab-

lished kinetic-MHD models $[4,6,9,10]$ to study energetic particle dynamics and multiscale kinetic-MHD phenomena.

Dominant magnetospheric dynamic behaviors such as magnetospheric substorms, recoonection and plasma transport processes at the magnetopause, and storm time MHD waves and plasma transport in the ring current region all involve complex multiscale kinetic-MHD 
coupling processes. We have also applied our kinetic-MHD models to study magnetospheric multiscale coupling processes such as kinetic effects on MHD waves in the magnetosphere and magnetopause and associated plasma transport. In particular, we have developed the theory of ballooning-mirror instability [11-13] that explains the multisatellite observation of field-aligned structure of compressional Pc 5 waves and their stability criteria in the ring current region. We have developed a kinetic-MHD theory of nonuniform drift-mirror modes to explain the mirror mode observation in the magnetosheath $[14,15]$. We have also developed a theory of the mode conversion of compressional MHD waves into kinetic Alfvén waves and associated plasma transport processes at the magnetopause [16]. These works involve particle kinetic effects on MHD phenomena and are all based on the kinetic-MHD formulation that is the theoretical foundation of our energetic particle physics.

To understand particle kinetic effects on MHD phenomena, we need to first understand the limitation of the MHD model. The single fluid MHD model treats the plasma as a conducting fluid and its major advantage is that the governing equations are much simpler than the kinetic equations and properly describe the global geometrical effects. The basic assumption of the ideal MHD model is that the plasma is frozen in the field line and moves with the $\vec{E} \times \vec{B}$ drift, and the parallel electric field is zero. In the resistive MHD limit the parallel electric field is proportional to the parallel current density through plasma resistivity. The plasma pressure follows the adiabatic pressure law through plasma convection as well as compression. The fundamental shortcomings of the MHD model are that (a) the magnetic drift velocity is assumed to be small in comparison with the $\vec{E} \times \vec{B}$ drift velocity and (b) kinetic effects such as finite particle Larmor radius, wave-particle resonances and particle trapping in a nonuniform magnetic field are ignored. Therefore, the basic assumptions of the MHD model can become invalid when particle kinetic effects are important. For example, energetic particles can significantly affect the MHD stability because their kinetic effects are vitally important due to high energy. For low frequency MHD modes with $\omega \ll \omega_{d}$, where $\omega_{d}$ is the particle magnetic drift frequency and is proportional to the particle energy, the energetic particle dynamics are no longer governed by the $\vec{E} \times \vec{B}$ drift, but rather 
by the magnetic ( $\nabla B$ and curvature) drift because the particle magnetic drift velocity is proportional to the particle energy. For MHD shear Alfvén waves with $\omega=\omega_{b}+\omega_{d}\left(\omega_{b}\right.$ is the energetic trapped particle bounce frequency), they can be driven unstable by energetic particles resonating with the background waves because $\omega_{b}$ is proportional to the particle velocity.

To take advantage of the simplicity of the MHD model and to properly take into account major kinetic effects of energetic particles, we have previously developed a kinetic-MHD model $[4,6,9]$. The plasma can be considered to consist of two components: (1) a low energy core component which has the major density fraction and (2) an energetic component which has low density, high energy and high $\beta$, and does not satisfy the MHD description. Each component can consist of more than one particle species. Instead of employing a full kinetic approach for all particle species, the kinetic-MHD model treats the low energy core plasma by the ideal MHD description and energetic particles by a kinetic approach such as the gyrokinetic equation [17] or Vlasov equation. The coupling between the dynamics of these two components of plasmas is through the plasma pressure in the momentum equation. Because the plasma resistivity and kinetic effects of the core component are neglected, the parallel electric field vanishes. The kinetic-MHD model optimizes both the physics content and the theoretical (analytical as well as numerical) effort, and properly accounts for the dynamics of high- $\beta$ plasma with pressure anisotropy in general magnetic field geometries. It is convenient for studying low frequency MHD type instabilities, wave propagation, and associated energetic particle transport.

In Sec. II we will review kinetic-MHD properties of plasma equilibria and present a dynamical kinetic-MHD model that incorporates particle kinetic effects. In Sec. III we present an application of the kinetic model to study the excitation of TAE instabilities by energetic ions in tokamaks. Then, in Sec. IV we demonstrate that the kinetic-MHD model can be successfully applied to study ballooning-mirror instabilities to understand the fieldaligned structure and instability threshold of compressional Pc 5 waves in the ring current region. Finally a summary and discussion is given in Sec. V. 


\section{A KINETIC-MHD MODEL INCORPORATING PARTICLE KINETIC EFFECTS}

To incorporate particle kinetic effects into MHD formulation we have developed a hybrid kinetic-MHD model $[4,6,9]$ for describing low-frequency MHD phenomena in high $\beta$ $(\beta \simeq O(1))$ anisotropic plasmas, which can be considered to consist of two components:

a low-energy core $(c)$ background component and an hot $(h)$ component with low density so that $n_{h} \ll n_{c}$, and $T_{h} \gg T_{c}$. The particle kinetic effects are mainly included in the plasma pressure through the momentum equation. The kinetic-MHD model is applicable to magnetized collisionless plasma systems where the parallel electric field effects are negligibly small.

\section{A. Kinetic-MHD Equilibrium}

To study particle kinetic effects on MHD phenomena, it is necessary to know the plasma equilibrium structure. If the plasma convection is small, the plasma equilibrium can be approximated by a static MHD equilibrium with anisotropic pressure, which is described by $\vec{J} \times \vec{B}=\nabla \cdot \boldsymbol{P}=\nabla P_{\perp}-\nabla \cdot\left[\left(P_{\perp}-P_{\|}\right) \hat{\mathbf{b}} \hat{\mathbf{b}}\right], \nabla \times \vec{B}=\vec{J}$, and $\nabla \cdot \vec{B}=0$, where $\hat{\mathbf{b}}$ is a unit vector along an equilibrium magnetic field line, and $\vec{J}, \vec{B}$, and $\boldsymbol{P}$ are the equilibrium current, magnetic field, and pressure tensor, respectively. In this paper the equations will be expressed in the rationalized MKS unit system.

Considering a general three-dimensional equilibrium with nested flux surfaces, the magnetic field can be expressed as $\vec{B}=\nabla \psi \times \nabla \alpha$, where $\psi$ is chosen as the magnetic flux function. Both $\psi$ and $\alpha$ are constant along magnetic field lines. The lines where surfaces of constant $\mathrm{y}$ and a intersect represent magnetic field lines. Note that $\psi$ must be a periodic function of toroidal angle $\phi$ in cylindrical $(R, \phi, Z)$ coordinate to ensure periodicity constraint. In terms of a flux coordinate system $(\psi, \Theta, \psi)$ with $\Theta$ being the generalized poloidal angle varying between 0 and $2 \pi, \alpha$ can be expressed as $\alpha=\phi-q(\psi) \Theta-\Delta(\psi, \Theta, \phi)$ without 
loss of generality, where $\Delta(\psi, \Theta, \phi)$ is periodic in both $\Theta$ and $\phi$. For tokamaks $q(\psi)$ is the safety factor. For a collisionless plasma the particle energy $\left(\mathcal{E}=v^{2} / 2\right)$ and the adiabatic invariants, magnetic moment $\left(\mu=v_{\perp}^{2} / 2 B\right)$ and the longitudinal invariant $\left(J_{\|}=\int d s v_{\|}\right)$, are constant during the drift motions, where $v_{\|}$and $v_{\perp}$ are the components of the velocity parallel and perpendicular to $\vec{B}$, respectively. The guiding-center equilibrium particle distribution function must have the form $F=F\left(\mathcal{E}, \mu, J_{\|}\right)$. In general, $J_{\|}=J_{\|}(\mathcal{E}, \mu, \psi, \alpha)$ and $F=F(\mathcal{E}, \mu, \psi, \alpha)$. If all particles on each field line share the same drift surface, where $\psi$ labels the drift surface, then $J_{\|}=J_{\|}(\mathcal{E}, \mu, \psi)$ and $F=F(\mathcal{E}, \mu, \psi)$. The guiding-center particle distributions $F=F(\mathcal{E}, \mu, \psi)$ can be either prescribed by an analytical form or obtained from the satellite measurements of the particle flux.

The equilibrium parallel and perpendicular pressures are given by

$$
\left(\begin{array}{c}
P_{\|} \\
P_{\perp}
\end{array}\right)=\sum_{j, \sigma_{\|}} 2 \pi M_{j} \int_{0}^{\infty} d \mathcal{E} \int_{0}^{\mathcal{E} / B} d \mu \frac{B F_{j}}{\left|v_{\|}\right|}\left(\begin{array}{c}
2(\mathcal{E}-\mu B) \\
\mu B
\end{array}\right),
$$

where the summation is over the particle species $j$ and $\sigma_{\|}$which represents the direction of particle velocity parallel to $\vec{B}$, and $M_{j}$ is the particle mass. The parallel velocity $v_{\|}$has the form $v_{\|}=\sigma_{\|} \sqrt{2(\mathcal{E}-\mu B)}$. By inspection, $P_{\perp}$ and $P_{\|}$are functions of $\psi$ and $B$ only.

It is convenient to introduce the functions

$$
\begin{aligned}
\sigma & \equiv 1-\frac{1}{B}\left(\frac{\partial P_{\|}}{\partial B}\right)_{\psi}, \\
\tau & \equiv 1+\frac{1}{B}\left(\frac{\partial P_{\perp}}{\partial B}\right)_{\psi} .
\end{aligned}
$$

If $\sigma>0$ and $\tau>0$ are satisfied everywhere in the plasma, the plasma is stable to the well-known MHD "firehose" and "mirror" instabilities, respectively [18]. The momentum balance equation parallel to the equilibrium magnetic field is given by

$$
\vec{B} \cdot \nabla P_{\|}=\left(P_{\|}-P_{\perp}\right) \hat{\mathrm{b}} \cdot \nabla B
$$

Making use of (2) - (4), the momentum balance equation perpendicular to the magnetic field is given by 


$$
\tau \nabla_{\perp}\left(B^{2} / 2\right)+\tilde{\nabla}_{\perp} P_{\perp}=\vec{\kappa} \sigma B^{2}
$$

where $\tilde{\nabla}=\nabla-\nabla B(\partial / \partial B)_{\psi}$, and $\overrightarrow{\boldsymbol{\kappa}}=\hat{\mathbf{b}} \cdot \nabla \hat{\mathbf{b}}$ is the magnetic field curvature. Note that the parallel momentum balance equation (Eq. (4)), is automatically satisfied if the particle distribution $F(\mathcal{E}, \mu, \psi)$ is used to compute $P_{\perp}$ and $P_{\|}$. From Eq. (4), $\sigma$ can be simplified as $\sigma=1+\left(P_{\perp}-P_{\|}\right) / B^{2}$.

In general, the equilibrium solutions can only be obtained by numerical solutions of Eqs. (4) and (5). However, to achieve analytical understanding of particle kinetic effects on MHD phenomena, simplified equilibrium solutions are usually employed.

\section{B. Kinetic-MHD Model}

Because the plasma usually has anisotropic pressure in both the space environment and large magnetic fusion devices, we consider the momentum equation with anisotropic pressure

$$
\rho \frac{d}{d t} \vec{V}=-\nabla \cdot \mathbf{P}+\vec{J} \times \vec{B}
$$

where $(d / d t)=(\partial / \partial t)+\vec{V} \cdot \nabla$ is the total time derivative, $\vec{V}$ is the fluid velocity, $\vec{B}$ is the magnetic field, $\mathbf{P}$ is the pressure tensor due to all particle species, and $\rho$ is the total plasma mass density. The total plasma pressure tensor can be expressed as $\mathbf{P}=P_{\perp} \mathbf{I}+\left(P_{\|}-\right.$ $\left.P_{\perp}\right) \vec{B} \vec{B} / B^{2}$, where $P_{\|}$and $P_{\perp}$ are the parallel and perpendicular pressures, respectively, and contain both the core and hot plasma pressures. The mass density continuity equation is given by

$$
\frac{d}{d t} \rho+\rho \nabla \cdot \vec{V}=0
$$

The Maxwell's equations hold: the Faraday's law, $\partial \vec{B} / \partial t=-\nabla \times \vec{E}$, where $\vec{E}$ is the electric field; the Ampere's law, $\vec{J}=\nabla \times \vec{B}$; and $\nabla \cdot \vec{B}=0$.

To close the above equations we need to prescribe the relation between the electric field and the fluid velocity as well as the dynamics of plasma pressure. If the core plasma finite Larmor radius (FLR) effect can be ignored, the Ohm's law, 


$$
\vec{E}+\vec{V} \times \vec{B}=0
$$

is usually employed so that the perpendicular fluid motion is mainly determined by the $\vec{E} \times \vec{B}$ motion. If the wave-particle resonances from core particle species can be ignored, the low energy core component can be reasonably well treated by the MHD description. Because the hot plasma density is much smaller than the core plasma density we employ the double-adiabatic pressure laws to relate the core plasma pressure to the plasma density;

$$
\frac{d}{d t}\left(\frac{P_{\| c} P_{\perp c}^{2}}{\rho^{5}}\right)=0
$$

and

$$
\frac{d}{d t}\left(\frac{P_{\perp c}}{\rho B}\right)=0
$$

If the kinetic effect of wave-particle resonances from all particle species is important, we shall obtain the parallel and perpendicular pressures from the particle distribution function $f$ given by

$$
\begin{gathered}
P_{\|}=\sum_{j} M_{j} \int d^{3} v v_{\|}^{2} f_{j} \\
P_{\perp}=\sum_{j} \frac{M_{j}}{2} \int d^{3} v v_{\perp}^{2} f_{j}
\end{gathered}
$$

where the summation in $j$ is over all particle species, $M$ is the particle mass, and $v_{\|}$and $v_{\perp}$ are the particle velocity parallel and perpendicular to the magnetic field $\vec{B}$, respectively.

If the plasma equilibrium scale length is much larger the particle gyroradii and the perturbation scale lengths, the low-frequency gyrokinetic formulation can be employed to describe the dynamics of all particle species for MHD phenomena. We consider waves with $\omega \ll \omega_{c i}, k_{\perp}>k_{\|}$and assume a WKB eikonal representation for perturbed quantities, i.e., $\delta f(\vec{x}, \vec{v}, t)=\delta f\left(s, \vec{k}_{\perp}, \vec{v}, t\right) \exp \left(i \int d \vec{x}_{\perp} \cdot \vec{k}_{\perp}\right)$, where $s$ is the distance along the equilibrium magnetic field. The perturbed particle distribution function for a given species is written as

$$
\delta f=\frac{q_{e}}{M} \frac{\partial F}{\partial \mathcal{E}} \Phi+\frac{q_{e}}{M B} \frac{\partial F}{\partial \mu}\left(\Phi-v_{\|} A_{\|}\right)+\left[g_{0}-\frac{q_{e}}{M B} \frac{\partial F}{\partial \mu}\langle\delta L\rangle\right] e^{i L_{0}}
$$


where $F$ is the equilibrium particle distribution function, $\langle\delta L\rangle=\left(\Phi-v_{\|} A_{\|}\right) J_{0}\left(k_{\perp} v_{\perp} / \omega_{c}\right)+$ $\left(v_{\perp} \delta B_{\|} / k_{\perp}\right) J_{1}\left(k_{\perp} v_{\perp} / \omega_{c}\right), L_{0}=\left(\vec{k}_{\perp} \times \vec{v}_{\perp} \cdot \vec{B}\right) / \omega_{c} B, \mathcal{E}=v^{2} / 2, \mu=v_{\perp}^{2} / 2 B, q_{e}$ is the particle charge, $\omega_{c}=q_{e} B / M c$, and $\Phi, A_{\|}$, and $\delta B_{\|}$are the electrostatic potential, vector potential and perturbed magnetic field parallel to the equilibrium magnetic field $\vec{B}$, respectively. $J_{0}$ and $J_{1}$ are the Bessel functions of order 0 and 1 , respectively. The nonadiabatic particle distribution $g_{0}$ is governed by the nonlinear gyrokinetic equation [17]

$$
\left[\frac{\partial}{\partial t}+\left(\vec{v}_{\|}+\vec{v}_{d} \cdot \nabla\right] g_{0}=-\left[\frac{q_{e}}{M} \frac{\partial F}{\partial \mathcal{E}} \frac{\partial}{\partial t}-\frac{\vec{B} \times \nabla\left(F+g_{0}\right)}{B^{2}} \cdot \nabla\right]\langle\delta L\rangle,\right.
$$

where $\vec{v}_{d}=\left(\vec{B} / B \omega_{c}\right) \times\left[\nabla(\mu B)+\vec{\kappa} v_{\|}^{2}\right]$ is the particle magnetic drift velocity. In the limit $k_{\perp} \rho_{i} \ll 1$, Eqs. (12) and (13) reduce to the guiding center limit with $L_{0}=0$ and $\langle\delta L\rangle=$ $\left(\Phi-v_{\|} A_{\|}\right)+\left(M c \mu \delta B_{\|} / q_{e}\right)$. Note that the resultant guiding center drift kinetic equation is valid even without the WKB eikonal representation of perturbed quantities.

Equations (6)-(13) form the basis of the gyrokinetic-MHD model that includes dominant particle kinetic effects. When a more accurate solution of $\delta f$ is required, such as in the magnetotail region where the particle Larmor radius is comparable to the equilibrium scale length, the Vlasov equation must be employed to solve $\delta f$ in order to couple to the MHD fluid equations.

In tokamak research, the kinetic-MHD model has been applied to study energetic particle physics such as the destabilization of TAE by energetic ions, and sawtooth (internal kink mode) stabilization and fishbone mode destabilization by energetic trapped ions. In particular, the TAE instability and the associated energetic transport is at present considered to be the most important energetic particle physics issue in the tokamak fusion research. In the magnetospheric physics research we have also applied our kinetic-MHD models to study magnetospheric multiscale coupling processes such as kinetic effects on MHD waves in the magnetosphere and magnetopause and associated plasma transport. In particular, we have successfully carried out a theory of ballooning-mirror instability destabilized by energetic protons to account for the structure and stability threshold of compressional Pc 5 waves in the ring current region obtained from the multi-satellite observation. In the fol- 
lowing we shall present kinetic-MHD theories for the TAE instability in tokamaks and for the ballooning-mirror instability in the magnetospheric ring current region.

\section{ENERGETIC PARTCILE EXCITATION OF TAE IN TOKAMAKS}

The study of energetic particle transport and kinetic effects on MHD modes is important for fusion reactor design and operation. Energetic ions, such as alpha particles produced in DT fusion reactions and energetic ions produced by auxiliary ICRF and N-NBI heating, are employed to heat the core thermal plasmas. Therefore, the behavior of energetic ions should be well understood. Any unanticipated loss of energetic/alpha particle power could result in reduction of plasma beta, serious wall damage, impurity influx, major operational control problems, or even a failure to sustain ignition. Recent NBI and ICRF experiments in large tokamaks [19-21] have shown that the TAE $[1,2]$ can be strongly unstable and cause the loss of up to half of the fast beam ions. This level of loss would be unacceptable in fusion reactors.

The TAE mode frequency is roughly given by $\omega \simeq \omega_{A} / 2$, where $\omega_{A}=V_{A} / q R, q$ is the safety factor, $R$ is the major radius, and $V_{A}$ is the Alfvén speed. The TAE can be driven unstable by energetic/alpha particles through wave-particle resonances by taping the free energy associated with the energetic particle pressure gradient. This mechanism can be understood by considering the linear power transfer from energetic particles to the TAE mode which is given by

$$
P_{0}=\int d^{3} v d^{3} x q_{e}^{2}\left(\frac{\omega_{*} F}{\omega}+T \frac{\partial F}{\partial \mathcal{E}}\right)\left(v_{\|} E_{\|}+\vec{v}_{d} \cdot \vec{E}_{\perp}\right)^{2} \delta\left(\omega-k_{\|} v_{\|}-\vec{k}_{\perp} \cdot \vec{v}_{d}\right),
$$

where $\omega_{*}$ is the diamagnetic drift frequency, $F$ is the particle distribution, $T$ is the particle temperature, $\mathcal{E}$ is the particle energy, $\vec{v}_{d}$ is the particle magnetic drift velocity, $k_{\|}$is the parallel wave vector, and $E_{\|}$and $\vec{E}_{\perp}$ are the parallel and perpendicular components of perturbed electric field, respectively. Physically, the $\partial F / \partial \mathcal{E}$ term gives the velocity space Landau damping, and the $\omega_{*}$ term is associated with the destabilizing pressure gradient. 
To destabilize the TAE mode, the instability drive associated with the energetic particle pressure gradient must overcome damping effects due to the continuum damping (if it exists), trapped electron collisional damping, and the velocity space Landau damping from all particle species. The TAE mode growth rate is typically on the order of $10^{-2} \omega_{A}$.

\section{A. Properties of TAE mode}

The TAE frequencies are located inside the shear Alfvén continuum gaps created due to the toroidal coupling of different poloidal harmonics [1,2]. If the toroidicity is neglected, the shear Alfvén continuous spectrum can be described in its cylindrical form $\omega^{2}=[(m-$ $\left.n q) V_{A} / q R\right]^{2}$, where $n$ and $m$ are the toroidal and poloidal mode numbers, respectively. The spectrum is continuous because the safety factor and the Alfvén velocity are functions of the minor radius. For example, the continuous spectra of the $(n, m)$ and $(n, m+1)$ modes cross at radial location $r_{0}$, where $q\left(r_{0}\right)=(m+1 / 2) / n$. The degeneracy is broken by the toroidal coupling effect to form a gap at $r_{0}$. The continuum gap structure can be computed by a variational principle with a Lagrangian functional [2]. For a large aspect ratio, low- $\beta$ tokamak equilibrium with circular outermost flux surface, the continuum gap boundary at $r_{0}$ is given to the first order in inverse aspect ratio $\left(\epsilon_{0}=r_{0} / R\right)$ by

$$
\omega_{ \pm}^{2}=\omega_{0}^{2}\left[1 \pm 2\left(r_{0} / R+\Delta^{\prime}\left(r_{0}\right)\right)\right]
$$

and the center of the continuum gap at $r=r_{0}$ is given to $O\left(\epsilon_{0}^{2}\right)$ by

$$
\omega_{0}^{2}=\left(V_{A} / 2 q R\right)^{2}\left[1+\frac{3}{2}\left(\frac{r_{0}}{R}+\Delta^{\prime}\right)^{2}+4 \frac{\Delta}{R}+{\frac{r_{0}}{R}}^{2}-2 \frac{r \Delta^{\prime}}{R}+2 g^{(2)}\right],
$$

where $\Delta(r)>0$ is the Shafranov shift of the non-concentric circular flux surfaces with $\Delta^{\prime}=d \Delta / d r>0$, and $g^{(2)}=-P / B^{2}+\int_{r}^{a} d r\left(2-r q^{\prime} / q\right) r / q^{2} R^{2}$ is the plasma beta and magnetic shear correction to the toroidal magnetic field. Therefore, $\omega_{0}^{2}$ will shift upward more than the widening of the gap as plasma beta increases. For TFTR supershot operations the Shafranov shift can be large with $\Delta / a \simeq 0.2$, and $\Delta^{\prime}$ can be as large as 0.3 . Thus, the finite pressure and aspect ratio effects on the continuum gap structure can be quite significant. 
The TAE modes had been shown $[1,2]$ to exist with discrete frequencies inside the continuum gap. For small (large) magnetic shear the TAE mode frequency is near the lower (upper) continuum gap boundary. Typically, for the $n=1 \mathrm{TAE}$ mode the $m=1$ and 2 poloidal harmonics are dominant components and peak near the $q=1.5$ surface. For the $n=2$ TAE mode the $m=2$ and 3 poloidal harmonics are dominant components and peak near the $q=1.25$ surface. The existence of TAE modes depends on plasma shaping, wall boundary condition, as well as the values and profiles of safety factor $q$, plasma beta $\beta$, and mass density $\rho$. For some equilibria, more than one TAE modes with different dominant poloidal components and radial structures can exist for each toroidal mode number. As the plasma beta increases or the magnetic shear decreases, the TAE frequency will move downward into the lower continuum and the TAE mode will experience continuum damping [22]. The beta limit above which TAE modes experience continuum damping will be lower for higher $n$ modes. For high- $n$ modes, the beta threshold is roughly given by $2 q^{2} R(d P / d r) / B^{2}=\hat{s}^{2} /(1+\hat{s})$ in the limit of small magnetic shear $\hat{s}=r q^{\prime} / q$. The ellipticity and triangularity of the plasma shaping will in general increase the critical beta as is the case for the beta limit of high- $n$ ballooning modes. The vacuum energy will increase the TAE frequency and the presence of wall reduces the critical beta.

\section{B. TAE Stability}

In large tokamals, the energetic ion energy typically ranges from about $100 \mathrm{keV}$ for NBI ions to a few $\mathrm{MeV}$ for fusion product ions (3.5 $\mathrm{MeV}$ for alpha particles). The tokamak magnetic field is usually around $5 \mathrm{~T}$. The thermal plasma density is on the order of $10^{14} \mathrm{~cm}^{-3}$, and the thermal plasma temperature is on the order of $10 \mathrm{keV}$ so that the thermal plasma $\beta$ is about a few percent. The energetic ion density is usually on the order of $10^{-2}$ of the thermal plasma density, and thus the hot particle $\beta$ is on the same order as (but usually less than) the thermal plasma $\beta$. Thus, the tokamak plasma basically consists of two com-

ponents: a core thermal component and a hot component with $n_{h}<<n_{c}$ and $T_{h} \gg>T_{c}$ so 
that $\beta_{h}<\beta_{c}$. Our kinetic-MHD model is therefore ideal for studying TAE modes and the associated energetic ion transport in tokamaks.

The linearized kinetic-MHD eigenmode equations for studying TAE stability can be treated in the limit of zero parallel electric field. Considering that perturbed quantities have a temporal dependence of $\exp (-i \omega t)$, and $\partial \vec{V} / \partial t=-i \omega \vec{\xi}$, where $\vec{\xi}$ is the plasma displacement vector, the linearized momentum equation is given by

$$
\rho \omega^{2} \vec{\xi}=\nabla \delta P_{c}+\nabla \cdot \delta \mathbf{P}_{h}+\delta \vec{B} \times(\nabla \times \vec{B})+\vec{B} \times(\nabla \times \delta \vec{B})
$$

where $\delta \vec{B}$ is the perturbed magnetic field, $\delta P_{c}$ is the perturbed core plasma pressure, and $\delta \mathbf{P}_{h}$ is the perturbed hot plasma pressure tensor. The Ohm's law and the Maxwell's equations become

$$
\delta \vec{B}=\nabla \times(\vec{\xi} \times \vec{B})
$$

and

$$
\vec{E}=i \omega \vec{\xi} \times \vec{B}
$$

Because the kinetic effect due to all particle species is important in determining the stability of TAE modes, we shall not make use of the adiabatic pressure law and instead compute all particle pressure responses from particle kinetic equations. By further assuming that the perpendicular wavelength is shorter than the parallel wavelength, which is in turn shorter than the equilibrium scale length $\left(k_{\perp} \gg k_{\|} \gg 1 / L\right)$, and the particle gyroradius is small compared to the perpendicular wavelength $\left(k_{\perp} \rho_{L} \ll 1\right)$ for all particle species, the perturbed particle distribution $\delta f$ is determined from the guiding-center drift kinetic equation. Then Eq. (12) can be written as

$$
\delta f=\vec{\xi}_{\perp} \cdot \nabla F-\frac{\mu}{B^{2}} \frac{\partial F}{\partial \mu} \vec{B} \cdot \delta \vec{B}+\hat{g} .
$$

The perturbed pressures for all particle species can be written as

$$
\left(\begin{array}{c}
\delta p_{\|} \\
\delta p_{\perp}
\end{array}\right)=\vec{\xi}_{\perp} \cdot \tilde{\nabla}\left(\begin{array}{c}
P_{\|} \\
P_{\perp}
\end{array}\right)+\frac{\vec{B} \cdot \delta \vec{B}}{B}\left(\frac{\partial}{\partial B}\right)_{\psi}\left(\begin{array}{c}
P_{\|} \\
P_{\perp}
\end{array}\right)+\left(\begin{array}{c}
\delta \hat{p}_{\|} \\
\delta \hat{p}_{\perp}
\end{array}\right),
$$


where $\tilde{\nabla}=\nabla-\nabla B(\partial / \partial B)_{\psi}$, and the particle kinetic effect is included in the nonadiabatic perturbed pressures $\delta \hat{p}_{\perp}$ and $\delta \hat{p}_{\|}$, which are given by

$$
\left(\begin{array}{c}
\delta \hat{p}_{\|} \\
\delta \hat{p}_{\perp}
\end{array}\right)=\sum_{j} M \int d^{3} v \hat{g}\left(\begin{array}{c}
2(\mathcal{E}-\mu B) \\
\mu B
\end{array}\right) .
$$

In the right-hand side of Eq. (21) the first term represents the convective derivative of plasma pressure, and the second term represents the compressional field effect associated with pressure nonuniformity along the field line. In the absence of parallel electric field the drift kinetic equation for the linearized nonadiabatic particle distribution function $\hat{g}$, Eq. (13), becomes

$$
\left[\frac{\partial}{\partial t}+\left(\vec{v} \|_{\|}+\vec{v}_{d}\right) \cdot \nabla\right] \hat{g}=\left[\frac{q_{e}}{M} \frac{\partial F}{\partial \mathcal{E}} \frac{\partial}{\partial t}-\frac{\vec{B} \times \nabla F}{B^{2}} \cdot \nabla\right] Y
$$

where $Y=-\left(i \vec{v}_{d} \cdot \vec{E} / \omega+M \mu \delta B_{\|} / q_{e}\right)$. In terms of a Fourier series representation the linearized nonadiabatic particle distribution function $\hat{g}$ has been derived for tokamaks [4-6] and will not be repeated here.

By taking an inner product of Eq. (17) with $\vec{\xi}^{\star}$ and integrating over the plasma volume with the assumption of a fixed conducting plasma boundary, we obtain a quadratic form given by

$$
D(\omega)=\delta W_{f}+\delta W_{k}-\delta K=0,
$$

where the inertial energy is given by

$$
\delta K=\omega^{2} \int d^{3} x \rho|\vec{\xi}|^{2}
$$

the total fluid potential energy due to both the core and hot components is

$$
\begin{aligned}
\delta W_{f}=\int & d^{3} x\left\{\left|\delta \vec{B}_{\perp}\right|^{2}+\left|\nabla \cdot \vec{\xi}_{\perp}+2 \vec{\kappa} \cdot \vec{\xi}_{\perp}\right|^{2} B^{2}\right. \\
& \left.\quad+\left(\vec{J} \cdot \vec{B} / B^{2}\right) \delta \vec{B} \times \vec{B} \cdot \vec{\xi}_{\perp}^{\star}-2\left(\vec{\kappa} \cdot \vec{\xi}_{\perp}^{\star}\right)(\vec{J} \times \vec{B}) \cdot \vec{\xi}_{\perp}\right\},
\end{aligned}
$$

and the particle kinetic potential energy is 


$$
\delta W_{k}=-\int d^{3} x\left[\delta \hat{p}_{\perp} \nabla \cdot \vec{\xi}^{\star}+\left(\delta \hat{p}_{\perp}-\delta \hat{p}_{\|}\right)\left(\vec{\kappa} \cdot \vec{\xi}_{\perp}^{\star}-\frac{\vec{B}}{B} \cdot \nabla \xi_{\|}^{\star}\right)\right]
$$

In deriving Eqs. (24)-(27), we have neglected the pressure anisotropy contribution from hot particles.

The TAE growth (or damping) rate is mainly determined by the wave-particle resonance contribution in $\delta W_{k}$. Because the TAE growth rate is very small in comparison with the real frequency, typically $|\gamma| \omega_{r} \mid \simeq 10^{-3}-10^{-2}$, where $\omega=\omega_{r}+i \gamma$, we can perform a perturbative analysis on the quadratic form, Eq. (24), to obtain the TAE mode frequency and growth rate:

$$
\omega_{r}^{2}=\left\{\delta W_{f}+\operatorname{Prin}\left[\delta W_{k}\right]\right\} / \delta K
$$

and

$$
\gamma=\operatorname{Res}\left[\delta W_{k}\right] / 2 \omega_{r} \delta K
$$

where $\left[\delta W_{k}\right]=\operatorname{Prin}\left[\delta W_{k}\right]+i \operatorname{Res}\left[\delta W_{k}\right]$ contains the principal part and the resonance contribution. The wave-particle resonances due to all particle species must be included in $\operatorname{Res}\left[\delta W_{k}\right]$. The core electron and ion distributions are taken as Maxwellians. In the numerical computation of Res $\left[\delta W_{k}\right]$ we have summed up many transit and bounce resonance terms to ensure its convergence. In reactors one would expect the $\alpha$-particle density to be $n_{\alpha} \propto P^{7 / 2}$. Thus the $\alpha$-particle pressure is usually quite localized around the magnetic axis with a typical scale length being roughly $10-40 \%$ of the minor radius.

A global non-variational stability kinetic-MHD stability code, the NOVA-K code $[6,23]$, has been developed to calculate the stability of the TAE modes based on the perturbative approach. The NOVA-K code calculates the destabilizing resonance contribution of energetic ions (fusion alphas, ICRF heated minority ions, and NBI ions) as well as the stabilizing effects of thermal particles by following the particle orbit in numerical tokamak equilibria. The collisional damping effect due to trapped electron is obtained by solving the bounceaveraged drift kinetic equation including the pitch angle scattering collisional operator, and 
no assumption on the ordering of $\nu_{e} / \omega$ is made, where $\nu_{e}$ is the electron collisional frequency. Numerical results are typically given in terms of the volume averaged energetic particle beta, $<\beta_{h}>$, and $\left(v_{h} / V_{A}\right)$ parameters. If the TAE mode does not suffer continuum damping,

the $\left\langle\beta_{h}>\right.$ threshold for TAE instability is typically about $10^{-4}-10^{-3}$ for $\left(v_{h} / V_{A}\right) \simeq$ 1. The TAE stability depends sensitively on actual tokamak equilibrium profiles and has been calculated for tokamak equilibria such as TFTR, JT-60U, JET, DIII-D, and ITER $[5,6,21,23-26]$.

One way to reduce or even stabilize the TAE instability is to control the plasma density and $q$ profiles so that the radial gap structure does not line up across the minor radius, and the TAE mode will experience continuum damping. The continuum damping effect on the TAE modes has been studied numerically with the NOVA-R resistive MHD code [27], and is typically less than $0.1 \%$ of the real frequency for resonance surface near the plasma edge.

\section{COMPRESSIONAL PC 5 WAVES AND BALLOONING-MIRROR INSTABILITIES IN THE RING CURRENT}

The kinetic-MHD model has also been successfully applied to study linear MHD instabilities in the ring current region of the magnetosphere. In particular, the ballooning-mirror instabilities have been identified to be responsible for the internally driven compressional Pc 5 waves [11-13] with wave period in the range of $150-600$ sec. Internally driven compressional Pc 5 waves usually have large azimuthal (east-west) mode numbers (typically 50-100) and are usually observed around the Earth's magnetic equator in the ring current region by satellites [28] during periods of enhanced ring current intensity and are associated with high$\beta$, anisotropic pressure plasmas with $P_{\perp}>P_{\|}$. Multisatellite (SCATHA, GOES 2, GOES 3, and GEOS 2) observations of a long lasting compressional Pc 5 wave event during November 14-15, 1979 [28-30] have revealed that the field-aligned structure of the compressional magnetic field $\delta B_{\|}$to be antisymmetric with respect to the Earth's magnetic equator with a full latitudinal range of about $20^{\circ}$ as shown in Fig. 1. The smaller transverse magnetic 
components, radial component $\delta B_{\psi}$ and azimuthal component $\delta B_{\phi}$, have a symmetric parity and their polarization varies with the magnetic latitude. The wave frequencies are about one order of magnitude smaller than the shear Alfvén frequency estimated from the measured plasma density and field-aligned wave structure near geosynchronous orbit. The internally driven transverse waves usually have low frequency and dominant radial polarization, and they are believed to have similar (but more extended) field-aligned wave structure as the compressional Pc 5 waves [31]. The antisymmetric $\delta B_{\|}$wave structure is now understood due to energetic trapped particle effects.

\section{A. Kinetic-MHD Eigenmode Equations}

To understand the internally driven compressional Pc 5 waves we have applied the kineticMHD model to study the ballooning-mirror instabilities $[9,11,12]$ in the magnetospheric ring current region, where the plasma pressure is anisotropic and is mainly contributed by energetic ions with energy larger than $1 \mathrm{keV}$. The plasma mass density (on the order of $1 \mathrm{~cm}^{-3}$ ) is mainly due to core (cold) ions with energy much less than $1 \mathrm{keV}$. Near the geosynchronous orbit, the magnetic field intensity is typically on the order of $100 \mathrm{nT}$, the hot ion (mainly proton) density is typically in the range of $10^{-2}-10^{-1} \mathrm{~cm}^{-3}$, and the hot ion energy is typically in the range of $10-10^{2} \mathrm{keV}$. Thus, the energetic ion $\beta$ is on the order of unity, and the core plasma $\beta$ is usually much smaller than unity and can be ignored.

Because $k_{\perp} \gg k_{\|}$for internally driven compressional Pc 5 waves, we shall consider a WKB waveform perpendicular to the ambient magnetic field so that $\nabla_{\perp}$ operates only on the perturbed quantities and $\nabla_{\perp}=i \vec{k}_{\perp}$. We will also assume that the parallel electric field vanishes so that the perturbed transverse magnetic field is related to the electrostatic potential $\Phi$ by $\delta \vec{B}_{\perp} \approx \nabla \times\left[\vec{B}\left(\vec{B} \cdot \nabla \Phi / \omega B^{2}\right)\right]$. The field-aligned kinetic-MHD eigenmode equations for $\hat{\Phi}(=\Phi / \omega)$ and $\delta B_{\|}$can be obtained with large azimuthal mode numbers from Eqs. (40), (41), and (45) in the paper by Cheng [9]. The compressional magnetic field equation is given by 


$$
\vec{B} \cdot \nabla\left(\frac{\sigma}{B^{2}} \vec{B} \cdot \nabla \delta B_{\|}\right)+\frac{\rho \omega^{2}}{B^{2}} \delta B_{\|}-k_{\perp}^{2}\left[\tau \delta B_{\|}-\frac{\vec{B} \times \widetilde{\nabla} P_{\perp} \cdot \vec{k}_{\perp}}{B^{3}} \hat{\Phi}+\frac{\delta \hat{p}_{\perp}}{B}\right]=0
$$

where $\hat{\Phi}(=\Phi / \omega)$, and $\widetilde{\nabla}=\nabla-\nabla B(\partial / \partial B)_{\psi}$, and the subscript $\psi$ represents the poloidal magnetic flux of a L-shell magnetic surface. Equation (30) indicates that the mirror mode can be destabilized by the combined effect of plasma $\beta$ and pressure anisotropy with $P_{\perp}>P_{\|}$. In the ideal MHD limit, mirror modes are unstable for $\tau<0$. The transverse magnetic field equation

$$
\vec{B} \cdot \nabla\left(\frac{\sigma k_{\perp}^{2}}{B^{2}} \vec{B} \cdot \nabla \hat{\Phi}\right)+\frac{\rho k_{\perp}^{2} \omega^{2}}{B^{2}} \hat{\Phi}+\frac{\vec{B} \times \overrightarrow{\boldsymbol{\kappa}} \cdot \vec{k}_{\perp}}{B^{2}}\left[\frac{\vec{B} \times \widetilde{\nabla} P_{\|} \cdot \vec{k}_{\perp}}{B^{2}} \hat{\Phi}+\sigma B \delta B_{\|}-\delta \hat{p}_{\|}\right]=0 .
$$

In the ideal MHD limit, the necessary condition is $\vec{\kappa} \cdot \nabla P>0$ for unstable ballooning modes and is $\sigma<0$ for the firehose instability. Note that kinetic effects due to hot trapped particles are included in the nonadiabatic perturbed pressures $\delta \hat{p}_{\perp}$ and $\delta \hat{p}_{\|}$. Equation (31) describes the ballooning mode which can be destabilized by the combined effect of the magnetic field curvature and the plasma pressure gradient. The coupling between the compressional and transverse magnetic field components, which determines the stability of the ballooningmirror instabilities, is due to finite perpendicular pressure gradient and the perturbed kinetic particle pressures.

\section{B. Quadratic Form}

A quadratic form can be derived from Eqs. (30) and (31) and provides significant insights to the stability properties. We assume that the perturbed quantities vanish at the end points of the field line, which means a perfectly reflecting boundary condition at the magnetosphereionosphere boundary. We first multiply Eq. (31) with $\hat{\Phi}^{*}$ and Eq. (30) with $\delta B_{\|}{ }^{*} / k_{\perp}^{2}$, and then sum up these two equations. After integrating along the field line with $d s / B$, where $s$ is the distance along the field line, and performing integration by parts, we obtain a quadratic form

$$
D(\omega)=\delta K-\delta W_{f}-\delta W_{k}=0,
$$


where the inertia energy is given by

$$
\delta K=\omega^{2} \int \frac{d s}{B} \frac{\rho}{B^{2}}\left[k_{\perp}^{2}|\hat{\Phi}|^{2}+\frac{\left|\delta B_{\|}\right|^{2}}{k_{\perp}^{2}}\right]
$$

The fluid potential energy is given by

$$
\begin{aligned}
\delta W_{f}=\int \frac{d s}{B}\left\{\frac{\sigma}{B^{2}} k_{\perp}^{2}|\vec{B} \cdot \nabla \hat{\Phi}|^{2}+\frac{\sigma}{B^{2} k_{\perp}^{2}}\left|\vec{B} \cdot \nabla \delta B_{\|}\right|^{2}\right. \\
\left.+\tau\left|\delta B_{\|}-\frac{\vec{k}_{\perp} \times \vec{B}}{\tau B^{3}} \cdot \tilde{\nabla} P_{\perp} \hat{\Phi}\right|^{2}-\tilde{\alpha}_{p}\left|\frac{k_{\perp} \hat{\Phi}}{L}\right|^{2}\right\},
\end{aligned}
$$

with

$$
\tilde{\alpha}_{p}=\left(\frac{\vec{k}_{\perp} \times \vec{B} \cdot \overrightarrow{\boldsymbol{\kappa}}}{k_{\perp} B}\right) \frac{\vec{k}_{\perp} \times \vec{B}}{k_{\perp} B^{3}} \cdot\left[\left(\frac{\sigma}{\tau}\right) \tilde{\nabla} P_{\perp}+\tilde{\nabla} P_{\|}\right] L^{2}
$$

and $L$ is the $L$-shell distance in the equatorial plane. The kinetic potential energy due to all species is

$$
\delta W_{k}=\int \frac{d s}{B}\left\{\left[\delta B_{\|}+\frac{\vec{k}_{\perp} \cdot \vec{B} \times \nabla\left(B^{2} / 2\right)}{B^{3}} \hat{\Phi}\right]^{*} \frac{\delta \hat{p}_{\perp}}{B}+\frac{\vec{k}_{\perp} \cdot \vec{B} \times \vec{\kappa}}{B^{2}} \hat{\Phi}^{*} \delta \hat{p}_{\|}\right\}
$$

In deriving the quadratic form we have made use of the equilibrium relation (Eq. (5)). In obtaining Eqs. (34) and (36) we have also ignored the contribution of the first two terms in Eq. (30). Thus the quadratic form is correct only in the limit that $\left(\rho \omega^{2} / B^{2}-k_{\|}^{2}\right) \ll \tau k_{\perp}^{2}$. Usually, $\tau$ is an increasing function as one moves along the field line from the equator toward the ionosphere. If $\tau$ is negative at the equator, it will be zero at some point along the field line, and the quadratic form will be incorrect. However, within the validity limit the quadratic form is useful in understanding the physics. In particular, $\delta W_{f}$ displays the various fluid free energy sources. If $\sigma>0$ (i.e., $1+\left(P_{\perp}-P_{\|}\right) / B^{2}>0$ ) and $\tau>0$ (i.e., $\left.1+(1 / B)\left(\partial P_{\perp} / \partial B\right)_{\psi}>0\right)$ are satisfied everywhere in the plasma, the magnetosphere is stable to the well-known MHD "firehose" and "mirror" instabilities, respectively [18]. The third term in Eq. (34) drives ballooning modes by pressure gradient in the bad curvature region where $\tilde{\alpha}_{p}>0$. 
The quadratic form (Eq. (32)), is not Hermitian, and there is no energy principle. However, if $\delta W_{k}$ is small compared with $\delta W_{f}$ and can be neglected, then Eq. (32) is an energy principle and the variational technique can be applied to obtain eigenvalue and eigenfunction. In the magnetospheric ring current region it had been shown [11] that $\delta W_{k}$ is small compared with $\delta W_{f}$ for $\hat{\Phi}$ and $\delta B_{\|}$with antisymmetric structure along the north-south symmetric equilibrium magnetic field lines in the low frequency limit with $\left(\omega-\omega_{d}\right) \ll \omega_{b}$, where $\omega_{d}=\left\langle\vec{k}_{\perp} \cdot \vec{v}_{d}\right\rangle$ is the bounce-averaged magnetic drift frequency and $\omega_{b}$ is the bounce frequency. In this limit, $\delta W_{k}$ is of the order of $\left(\left(\omega-\omega_{d}\right) / \omega_{b}\right)$ smaller than $\delta W_{f}$ since the contributions from the $\left(\omega-\omega_{d}\right)=0$ magnetic drift-bounce resonance vanishes. Therefore, if $\delta W_{k}$ can be neglected, two parameters, $\tilde{\alpha}_{p}$ and $\tau$, will determine the MHD stability. In the limit that the transverse and compressional magnetic field components decouple, it is possible that the ballooning instability can occur with perturbation localized around the region where $\tilde{\alpha}_{p}>0$, and the mirror mode can be unstable with perturbation localized around the region where $\tau<0$. In general, the transverse and compressional perturbed magnetic field components couple, and the stability properties will be modified.

In the limit $\left(\omega-\omega_{d}\right) \approx \omega_{b}$ the drift-bounce resonance can contribute to $\delta W_{k}$, it was proposed that the drift Alfvén ballooning-mirror instability can be destabilized by hot particle pressure gradient via the magnetic drift-bounce resonance in the form of compressional magnetic field with antisymmetric field-aligned structure [32]. The relationship between the ballooning-mirror mode and the drift Alfvén ballooning-mirror mode is still not yet resolved. It is conjectured that the drift Alfvén ballooning mirror instability may have a slightly lowerpressure anisotropy threshold than the mirror instability. However, wave resonance with all particle species must be included in the calculation before a definite answer on the stability threshold can be concluded. 
C. Energetic Trapped Particle Stabilization of Symmetric Ballooning-Mirror Modes

Kinetic effects in $\delta \hat{p}_{\|}$and $\delta \hat{p}_{\perp}$ are associated with energetic trapped particles and play an essential role in determining the stability and structure of the ballooning-mirror mode. Without the hot particle kinetic effects MHD modes with symmetric structure of parallel perturbed magnetic field, $\delta B_{\|}$, and electrostatic potential, $\Phi$, along the north-south ambient magnetic field would be more unstable than the antisymmetric mode. However, if energetic trapped particle kinetic effects are included, the symmetric ballooning-mirror mode can be completely stabilized. This process can be understood by considering the particle dynamics in a MHD perturbation. If the ballooning-mirror instability frequency is smaller than the energetic trapped particle magnetic drift frequency (which is much smaller than the bounce frequency) energetic trapped particles experience a finite bounce-averaged wave structure and their kinetic pressure response from $\delta \hat{p}_{\|}$and $\delta \hat{p}_{\perp}$ cancels with their fluid pressure response so that the symmetric mode is stabilized. Physically, the energetic trapped particles precess rapidly across the magnetic field, and their motion becomes rigid with respect to low frequency symmetric MHD perturbations.

To show the energetic particle stabilization of symmetric ballooning-mirror modes, we consider modes near marginal stability. By assuming that $\left|\sigma k_{\|}^{2}\right| \ll\left|\tau k_{\phi}^{2}\right|$, and from Eq. (30), we have $\delta B_{\|} \approx-\left(\vec{B} \times \tilde{\nabla} P_{\perp} \cdot \nabla \hat{\Phi} / B^{2}+\delta \hat{p}_{\perp}\right) / \tau B$. Then Eq. (31) reduces to

$$
\begin{aligned}
& \vec{B} \cdot \nabla\left(\frac{\sigma k_{\perp}^{2}}{B^{2}} \vec{B} \cdot \nabla \hat{\Phi}\right)+\frac{\rho k_{\perp}^{2}}{B^{2}} \hat{\Phi}+\frac{\vec{B} \times \vec{\kappa} \cdot \vec{k}_{\perp}}{B^{2}} \\
& {\left[\left(\frac{\vec{B} \times \tilde{\nabla} P_{\|}}{B^{2}}+\frac{\sigma \vec{B} \times \tilde{\nabla} P_{\perp}}{\tau B^{2}}\right) \cdot \vec{k}_{\perp} \hat{\Phi}+\delta \hat{p}_{\|}+\frac{\sigma \delta \hat{p}_{\perp}}{\tau}\right]=0 . }
\end{aligned}
$$

In the limit of small particle gyroradius and small wave and magnetic precessional drift frequencies, we can neglect contributions that are of the order of $\left(\left(\omega-\omega_{d}\right) / \omega_{b}\right)$, where $\omega_{b}$ is the particle bounce frequency, and the nonadiabatic energetic trapped particle pressure perturbations are then given by [11]

$$
\left(\begin{array}{c}
\delta \hat{p}_{\| \mid} \\
\delta \hat{p}_{\perp}
\end{array}\right)=\sum_{j} \int d^{3} v \frac{\left(-\omega \partial F / \partial \mathcal{E}-\omega_{*} F / \mathcal{E}\right.}{\omega-<\omega_{d}>}<Z>\cdot\left(\begin{array}{c}
2(\mathcal{E}-\mu B) \\
\mu B
\end{array}\right)+\mathcal{O}\left(\omega / \omega_{b}\right),
$$


where $Z=q_{e} \omega_{d} \hat{\Phi}+M \mu \delta B_{\|}$, the trapped particle bounce average of $Z$ is given by $\langle Z>=$ $\oint d s Z /\left|v_{\|}\right| / \oint d s /\left|v_{\|}\right|$with the integration along the trapped particle trajectory in one bounce period, $\omega_{*}=M \mathcal{E}_{0}\left(\vec{B} \times \nabla \ln F \cdot \vec{k}_{\perp}\right) / q_{e} B^{2}$, and $M \mathcal{E}_{0}$ is a typical particle energy.

We consider low-frequency MHD modes with symmetric $\hat{\Phi}$ and $\delta B_{\|}$structures. To carry out the velocity integration in Eq. (38), we further assume $\omega \ll \omega_{*},\left\langle\omega_{d}\right\rangle$, and $\langle Z\rangle \approx$ $\left.q_{e}<\omega_{d}\right\rangle \hat{\Phi}$ so that in the fast bounce timescale trapped particles experience the local wave structure. These approximations are reasonably good because the $\delta B_{\|}$term is small in comparison with the potential term in $Z$, and the average wave perturbation that deeply trapped particles experience in a fast bounce period is not very much different from the local values for symmetric $\hat{\Phi}$ and $\delta B_{\|}$waves. Equation (38) can be integrated to give

$$
\left(\frac{\vec{B} \times \tilde{\nabla} P_{\|}}{B^{2}}+\frac{\sigma \vec{B} \times \tilde{\nabla} P_{\perp}}{\tau B^{2}}\right) \cdot \vec{k}_{\perp} \hat{\Phi}+\delta \hat{p}_{\|}+\frac{\sigma}{\tau} \delta \hat{p}_{\perp} \approx 0,
$$

which shows that the fluid magnetic field curvature-pressure gradient driven term in the ballooning mode equation (Eq. (37)), is canceled by the nonadiabatic hot trapped particle pressure response. Therefore symmetric $\hat{\Phi}$ and $\delta B_{\|}$eigenmodes are stable. Physically, the hot trapped ion stabilization of ultra low frequency $\left(\omega \ll \omega_{*},<\omega_{d}>\right)$ MHD modes with symmetric $\hat{\Phi}$ and $\delta B_{\|}$structure is due to the fact that the hot trapped ion dynamic is no longer governed by the $\vec{E} \times \vec{B}$ drift, but rather by the magnetic $\nabla B$ and curvature drifts. To perturb the hot trapped ion pressure, the hot ion precession orbit must be perturbed through the perturbed electric and magnetic fields. Since the hot trapped ions precess very rapidly across the $\vec{B}$ field, their motion becomes very rigid with respect to the ULF symmetric MHD perturbation. Equation (39) indicates that the nonadiabatic hot trapped ion pressure changes through the perturbed perpendicular magnetic field (through electrostatic potential $\Phi)$ so that their total pressure perturbation due to both the fluid and kinetic responses is zero (see Eq. (21) in the limit of small $\delta B_{\|}$). 


\section{Field-Aligned Structure of Perturbed Magnetic Field}

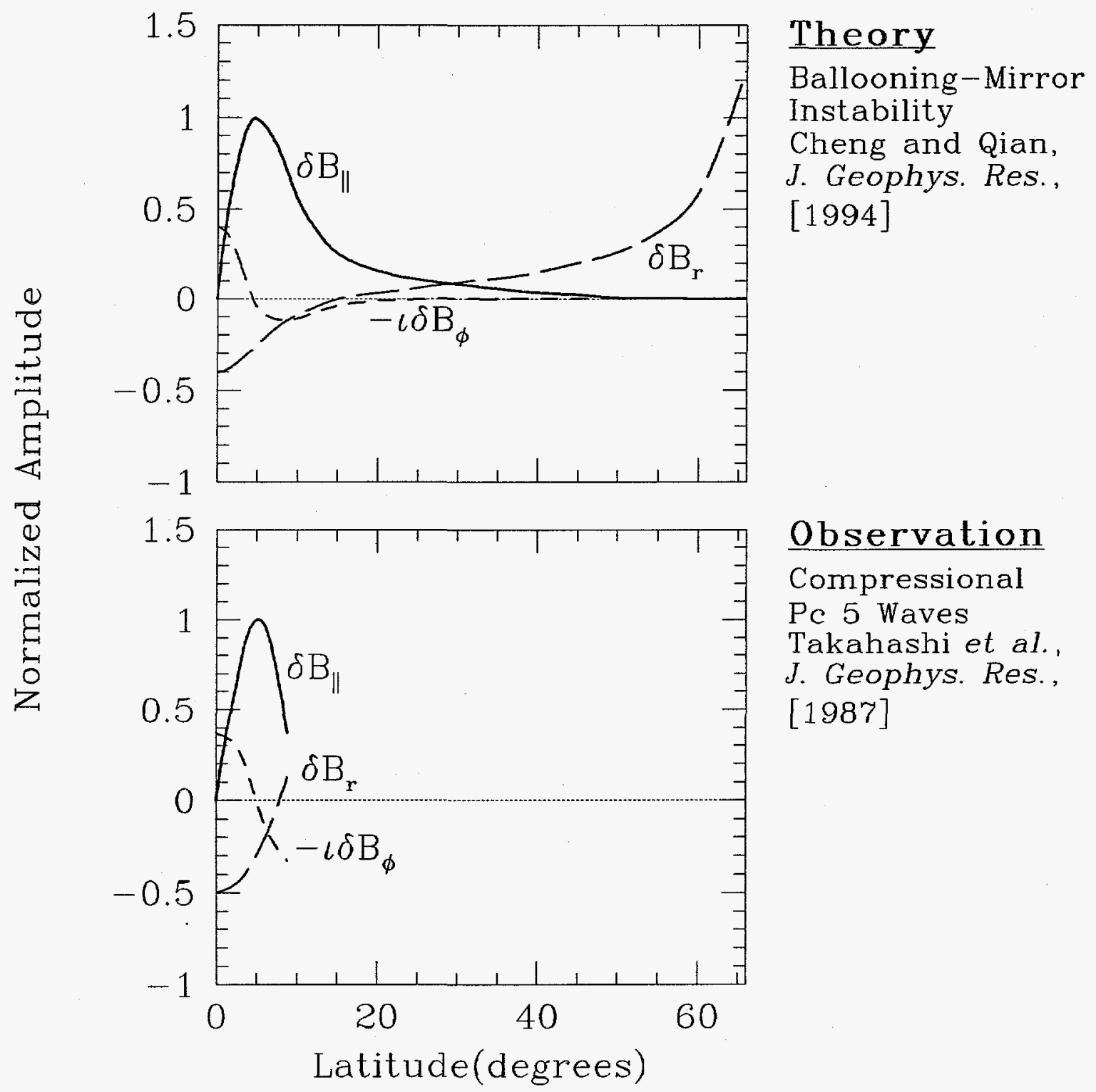

FIG. 1. A comparison of the field-aligned structure of the perturbed magnetic field between a theoretical solution of an antisymmetric ballooning-mirror mode and the multi-satellite (SCATHA, GOES 2, GOES 3, GEOS 2) observation of a long lasting compressional Pc 5 wave event during November 14-15, 1979. 


\section{Antisymmetric Ballooning-Mirror Instabilities}

On the other hand, from Eq. (38) the nonadiabatic perturbed hot trapped ion pressures responding to low frequency antisymmetric $\hat{\Phi}$ and $\delta B_{\|}$perturbations are vanishingly small because the trapped ion bounce orbit average of antisymmetric waves vanishes on the faster bounce timescale. This is due to the fact that the hot trapped ions bounce very rapidly along the north-south symmetric field line and the nonadiabatic (kinetic) hot trapped ion pressure response from antisymmetric $\hat{\Phi}$ and $\delta B_{\|}$perturbations in the northern hemisphere cancels that from the southern hemisphere. From Eq. (37) the hot ion fluid pressure response survives and determines the ballooning-mirror mode stability. Therefore, the antisymmetric ballooning-mirror modes have the lowest critical $\beta$ threshold for instability, and we expect that the ULF waves observed in the ring current region should have antisymmetric $\hat{\Phi}$ and $\delta B_{\|}$field-aligned structures. The conclusion was also previously pointed out $[9,11,12]$.

However, for antisymmetric modes the energetic trapped particle kinetic pressure response from the northern hemisphere cancels with that from the southern hemisphere in a bounce period so that both $\delta \hat{p}_{\|}$and $\delta \hat{p}_{\perp}$ vanish, and the instability $\beta$ threshold is determined by the energetic particle fluid free energy. For $\left(P_{\perp} / P_{\| 1}\right) \geq\left(1+1 / \beta_{\perp}\right)$, the field-aligned structure of the antisymmetric ballooning-mirror instability agrees with the multi-satellite observation of compressional Pc 5 wave structure [28-30]. In Fig. 1 we present a comparison of the field-aligned structure of the perturbed magnetic field between a theoretical solution of an antisymmetric ballooning-mirror mode and the multi-satellite (SCATHA, GOES 2, GOES 3, GEOS 2) observation of a long lasting compressional Pc 5 wave event during November 14-15, $1979[29,30]$. The numerical solution was obtained for bi-Maxwellian plasmas in a dipole magnetic field with the equatorial parameters: $P_{\perp} / P_{\|}=2, \beta_{\|}=0.575(\tau=-0.149)$, $\partial \ln P / \partial \ln L=-5$, and $L=6.6$. The agreement between the observation and our theory strongly suggests that this particular multi-satellite observation is related to marginally unstable ballooning-mirror modes with $\tau$ value close to zero, where $\tau=1+\beta_{\perp}\left(1-P_{\perp} / P_{\|}\right)$.

Figure 2 shows the theoretical stability boundaries of the antisymmetric ballooning- 


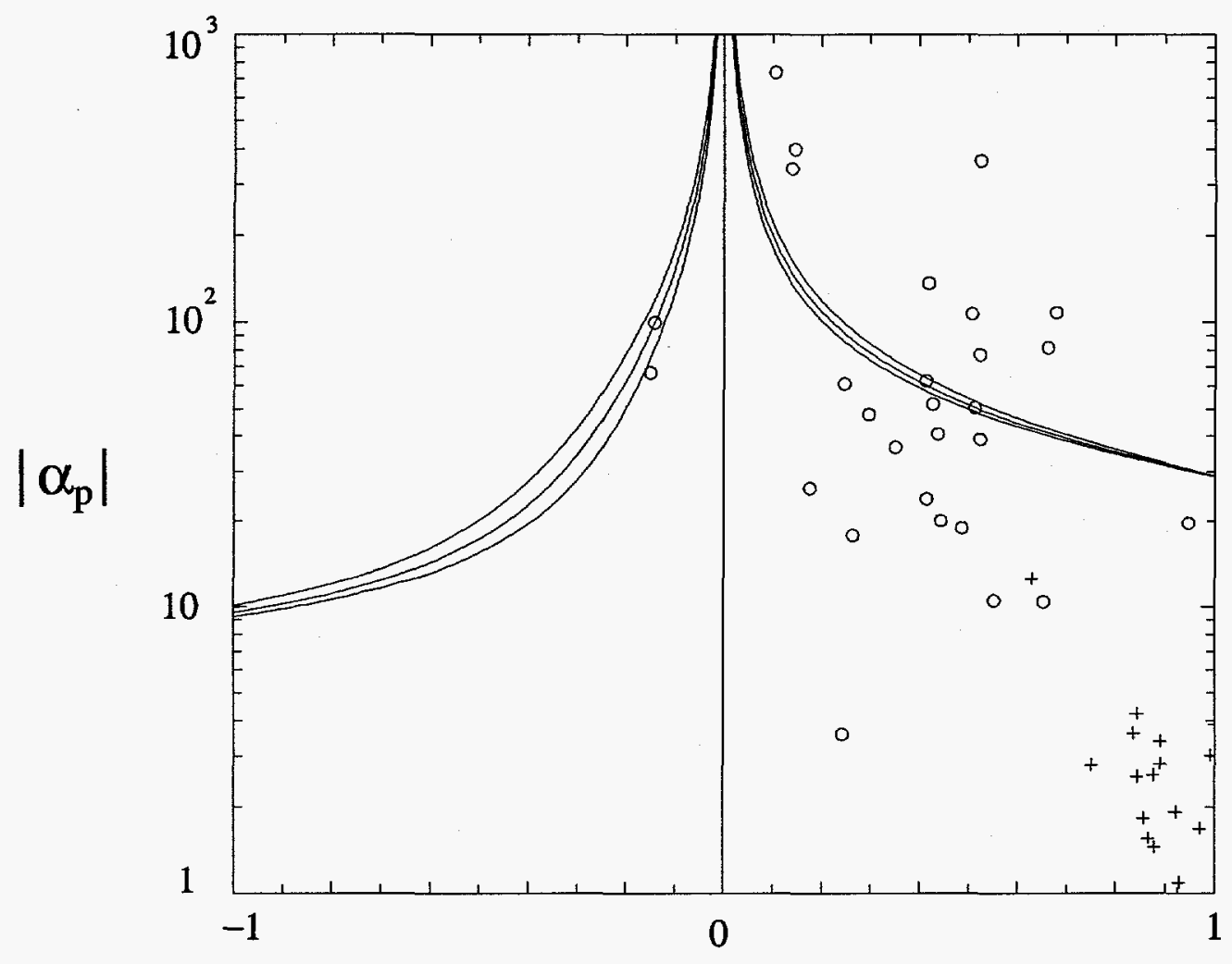

\section{$\tau$}

FIG. 2. A comparison of the theoretical stability boundaries of the antisymmetric ballooning-mirror modes for different $\partial \ln P / \partial \ln L$ values in the equatorial $\left(\tau, \alpha_{p}\right)$ space against "observed" values computed from the AMPTE/CCE particle data acquired with the ion charge-energy-mass spectrometer (CHEM) for 10 Pc 4-5 compressional and transverse wave events.

mirror modes for different $\partial \ln P / \partial \ln L$ values in the equatorial $\left(\tau, \alpha_{p}\right)$ space, where $\alpha_{p}$ represents the ballooning instability parameter and is given by

$$
\alpha_{p}=\frac{k_{\phi}^{2}}{k_{\perp}^{2}}\left(\kappa_{c} L\right)\left[\frac{\sigma}{\tau} \frac{P_{\perp}}{B^{2}} \frac{\partial \ln P_{\perp}}{\partial \ln L}+\frac{P_{\|}}{B^{2}} \frac{\partial \ln P_{\|}}{\partial \ln L}\right],
$$

where $\kappa_{c}$ is the normal magnetic field curvature across the L-shell. Note that $\alpha_{p}$ and $\tau$ are not linearly independent and for $\tau<0$ the $\alpha_{p}$ threshold is also negative, and we have plotted absolute value of $\alpha_{p}$ in Fig. 2 for $\tau<0$. Above the marginal stability boundary curves the antisymmetric ballooning-mirror modes are unstable. The marginal stability boundary curves in the equatorial $\left(\tau, \alpha_{p}\right)$ space are very close to each other even for $100>\partial \ln P / \partial \ln L>1$. This probably holds even for different type of particle velocity distributions. This property 
is useful when we compare theoretical calculations with satellite observations where information on $\partial \ln P / \partial \ln L$ and particle velocity distribution either is unavailable or cannot be obtained accurately. To test the theoretical antisymmetric ballooning-mirror instability threshold against observations we have computed values of $\tau$ and $\alpha_{p}$ from the AMPTE/CCE particle data acquired with the ion charge-energy-mass spectrometer (CHEM) for ten Pc 45 wave events [13]. In Fig. 2 we also plotted the "observed" values of $\tau$ and $\alpha_{p}$ against the theoretical stability threshold. The symbol " $\mathrm{O}$ " represents compressional wave events and the symbol "t" represents transverse wave events. When there is no wave activity the values of $\tau$ and $\alpha_{p}$ tend to be well below the antisymmetric ballooning-mirror instability thresholds. The transverse and compressional wave events clearly occupy different domains in the $\left(\tau, \alpha_{p}\right)$ space. For compressional wave events the values of $\tau$ and $\alpha_{p}$ are either near or above the theoretical stability boundary curves of the antisymmetric ballooning-mirror modes with $\tau \leq 0.6$ and $O(10) \leq \alpha_{p} \leq O\left(10^{3}\right)$. The transverse waves tend to occur when $\tau$ is close to unity and $1 \leq \alpha_{p} \leq O(10)$, which are at least one order of magnetitude below the theoretical antisymmetric ballooning-mirror instability threshold.

Therefore, we have demonstrated that internally driven compressional Pc 5 waves are caused by antisymmetric ballooning-mirror modes and can be successfully studied by our kinetic-MHD model $[9,12]$. They represent genuine multiscale kinetic-MHD phenomena; fast time scale trapped particle motion strongly couples to slow time scale MHD modes. We emphasize that the ideal MHD theory predicts an opposite result from our kinetic-MHD theory which is in close agreement with the observations.

\section{SUMMARY AND DISCUSSION}

In this paper we have presented a kinetic-MHD model for studying low frequency multiscale phenomena in a two component plasma (a low-energy core component and a highenergy low-density component) by taking into account the simplicity of the single fluid MHD model and major particle kinetic effects. If we can ignore the finite Larmor radius (FLR) 
effect of the low-energy core plasma, the Ohm's law is used to relate the core plasma fluid velocity with the electric field. If the wave-particle resonance effects of the low-energy core plasma can be ignored, the pressure of the low-energy core plasma can be described by the double-adiabatic pressure law. If the wave-particle resonance effect of the low-energy core plasma is important, a drift kinetic equation can be employed to describe the core plasma dynamics. For energetic particles a kinetic approach such as the gyrokinetic equation must be employed to describe the particle dynamics. The coupling between the kinetic particle dynamics and the MHD fluid is through the plasma pressure in the momentum equation. The major advantage of the kinetic-MHD model is that important kinetic effects can be accurately described with a minimum modification to the single fluid MHD equations. We note that important particle kinetic effects such as FLR effects of energetic particles and resonant wave-particle interactions of all particle species are included.

Based on the kinetic-MHD model we have successfully studied the energetic particle excitation of TAEs in tokamak plasmas. By employing a perturbative approach we have developed a global non-variational kinetic-MHD stability code, the NOVA-K code, which includes wave-particle resonance effects for all particle species to determine the TAE stability. The numerical results were able to explain TAE activities observed in major tokamaks such as TFTR, JT-60U, DIII-D, and JET. The NOVA-K code has also been used for studying the TAE stability issue in ITER.

The kinetic-MHD model has also been successfully applied to study ballooning-mirror instabilities to understand the field-aligned structure and instability threshold of compressional $\mathrm{Pc} 5$ waves in the ring current region. For ballooning-mirror instabilities in the ring current region, the plasma pressure is mainly contributed by hot ions and the core ion kinetic effects can be neglected. The hot trapped particle effects stabilize ballooning-mirror modes with symmetric field-aligned $\delta B_{\|}$structure because hot ions precess very rapidly across the magnetic field and their motion becomes rigid with respect to symmetric ballooning-mirror modes. On the other hand, the bounce motion of hot trapped ions is very rapid and their kinetic effects average out in a bounce period for antisymmetric ballooning-mirror modes. 
Thus, the threshold of antisymmetric ballooning-mirror instabilities is determined by the MHD fluid free energy. The ballooning-mirror instability represents a genuine example of multiscale kinetic-MHD phenomena; fast time scale trapped particle motion strongly couples to slow time scale MHD modes. We emphasize that the MHD theory would predict an opposite result from our kinetic-MHD theory which is in close agreement with the observations.

Finally, it is worthwhile to point out that we have recently extended the kinetic-MHD model presented in this paper by developing a generalized single fluid MHD model that treats core plasma FLR effects [10]. The major advantage of the new kinetic-MHD model is that important kinetic effects can be accurately described with minimum modification to the single fluid MHD equations. The generalized MHD model for the core plasma includes the parallel electric field, modified perpendicular velocity and gyroviscosity tensor due to core ion FLR effects, which are neglected in the single fluid MHD description. The perturbed core plasma density, velocity and pressure tensor (consisting of the diagonal pressure and gyroviscosity) are determined from approximate solutions of the gyro-kinetic equation. From the quasineutrality condition, we obtain the parallel electric field, which arises from the ion polarization drift and parallel electron inertia effects. The generalized Ohm's law contains core ion FLR effects on the parallel electric field and the perpendicular fluid velocity. The kinetic-MHD model is closed by generalized pressure laws, which contain kinetic effects from both the core and energetic plasma components. We note that the generalized MHD equtions properly retain important MHD effects such as background density, temperature and magnetic field gradients; magnetic field curvature; large plasma $\beta$; and pressure anisotropy. We also note that important energetic particle kinetic effects such as FLR; resonant wave-particle interactions; and bounce resonance are added. These kinetic effects are essential when describing multiscale coupling processes. 


\section{ACKNOWLEDGMENTS}

This work is supported by the NSF grant ATM-9523331 and DoE Contract No. DEAC02-76-CHO3073.

\section{DISCLAIMER}

This report was prepared as an account of work sponsored by an agency of the United States Government. Neither the United States Government nor any agency thereof, nor any of their empleyees, makes any warranty, express or implied, or assumes any legal liability or responsibility for the accuracy, completeness, or usefulness of any information, apparatus, product, or process disclosed, or represents that its use would not infringe privately owned rights. Reference herein to any specific commercial product, process, or service by trade name, trademark, manufacturer, or otherwise does not necessarily constitute or imply its endorsement, recommendation, or favoring by the United States Government or any agency thereof. The views and opinions of authors expressed herein do not necessarily state or reflect those of the United States Government or any agency thereof. 


\section{REFERENCES}

[1] C. Z. Cheng, L. Chen, and M. S. Chance, Annals Phys. 161, 21 (1985).

[2] C. Z. Cheng and M. S. Chance, Phys. Fluids 29, 3695 (1986).

[3] C. Z. Cheng, G. Y. Fu, and J. W. V. Dam, in Proc. of Workshop on Theory of Fusion Plasmas, Internal School of Plasma Physics, Chexbres, Switzerland, 3-7 October, 1988 (ed. by J. Vaclavik, F. Troyan and E. Sindoni, Societa Italiana Di Fisica, Italy, 1989), pp. 259-270.

[4] C. Z. Cheng, Phys. Fluids B 2, 1427 (1990).

[5] C. Z. Cheng, Phys. Fluids B 3, 2463 (1991).

[6] C. Z. Cheng, Phys. Reports 211, 1 (1992).

[7] R. B. White, P. H. Rutherford, P. Colestock, and M. N. Bussac, Phys. Rev. Lett. 60, 2038 (1988).

[8] L. Chen, R. B. White, and M. N. Rosenbluth, Phys. Rev. Lett. 52, 1122 (1984).

[9] C. Z. Cheng, J. Geophys. Res. 96, 21,159 (1991).

[10] C. Z. Cheng and J. R. Johnson, in Physics of Space Plasmas (1995) (MIT Center For Theoretical Geo/Cosmos Plasma Physics, Cambridge, MA, 1996), pp. 127-145.

[11] C. Z. Cheng and C. S. Lin, Geophys. Res. Lett. 14, 884 (1987).

[12] C. Z. Cheng and Q. Qian, J. Geophys. Res. 99, 11,193 (1994).

[13] C. Z. Cheng, Q. Qian, K. Takahashi, and A. T. Y. Lui, J. Geomag. Geoelectr. 46, 997 (1994).

[14] J. R. Johnson and C. Z. Cheng, in Physics of Space Plasmas (1995) (MIT Center For Theoretical Geo/Cosmos Plasma Physics, Cambridge, MA, 1996), pp. 261-279.

[15] J. R. Johnson and C. Z. Cheng, (1996), accepted for publication in J. Geophys. Res. 
[16] J. R. Johnson and C. Z. Cheng, (1997), submitted to Geophys. Res. Lett.

[17] E. A. Frieman and L. Chen, Phys. Fluids 25, 502 (1982).

[18] H. Grad, in Magneto-Fluid and Plasma Dynamics, Symp. in Appl. Math., Am. Math. Soc., New York (PUBLISHER, ADDRESS, 1967), Vol. 18, p. 162.

[19] K. L. Wong et al., Phys. Rev. Lett. 66, 1874 (1991).

[20] W. W. Heidbrink et al., Nucl. Fusion 31, 1635 (1991).

[21] H. Kimura et al., Phys. Lett. A 199, 86 (1995).

[22] G. Y. Fu and C. Z. Cheng, Phys. Fluids B 2, 985 (1990).

[23] G. Y. Fu, C. Z. Cheng, and K. L. Wong, Phys. Fluids B 5, 4040 (1993).

[24] C. Z. Cheng et al., in Plasma Physics and Controlled Nuclear Fusion Research 1992, Vol. 2 (International Atomic Energy Agency, Vienna, 1994), pp. 51-70.

[25] C. Z. Cheng et al., in Plasma Physics and Controlled Nuclear Fusion Research 1994, Vol. 3 (International Atomic Energy Agency, Vienna, 1996), pp. 373-389.

[26] G. Y. Fu et al., Phys. Rev. Lett. 75, 2336 (1995).

[27] T. R. Harley, C. Z.Cheng, and S. C. Jardin, J. Comput. Phys. 103, 43 (1992).

[28] K. Takahashi, Adv. Space Res. 8, 427 (1988).

[29] K. Takahashi, J. F. Fennell, E. Amata, and P. R. Higbie, J. Geophys. Res. 92, 5857 (1987).

[30] K. Takahashi, C. Z. Cheng, R. W. McEntire, and L. M. Kistler, J. Geophys. Res. 95, 977 (1990).

[31] M. J. Engebretson et al., J. Geophys. Res. 93, 12795 (1988).

[32] L. Chen and A. Hasegawa, J. Geophys. Res. 96, 1503 (1991). 